\title{
Complete Ankylosis in Longterm HLA-B27-negative Psoriatic Arthritis
}

MARC FRERIX, MD; ULF MÜLLER-LADNER, MD; UWE LANGE, MD, Department of Rheumatology and Clinical Immunology, Kerckhoff-Clinic Bad Nauheim, Justus-Liebig University Giessen, Bad Nauheim, Germany. Address correspondence to Dr. Frerix; E-mail: m.frerix@kerckhoff-klinik.de; J Rheumatol 2012;39:2177-8; doi:10.3899/jrheum.120641

Severe axial and peripheral involvement in HLA-B27-negative psoriatic arthritis (PsA) remains very rare.

In 2011, an 82-year-old woman who had had PsA since 1973 was referred to our department. She had plaque psoriasis at elbows, knees, and scalp since 1972, and the first diagnosis of PsA was in 1973. In 1974, she received 15 injections with gold-sodium thiomalate, but this was terminated because of lack of efficacy. In 1978, tenosynovectomy procedures of the right digiti II-V were performed.

In the following years, she reported predominantly pain of ankles and wrists, and progressive loss of flexibility of the vertebral column. Because of continuing pain, she was given celecoxib $200 \mathrm{mg} / \mathrm{bid}$ and subsequently diclofenac 75 $\mathrm{mg} / \mathrm{bid}$ for the last 9 years, without satisfactory benefit. A rheumatologist had not seen her during these years since first diagnosis. At time of first visit to our clinic she presented with severe plaque psoriasis, psoriatic nail manifes-

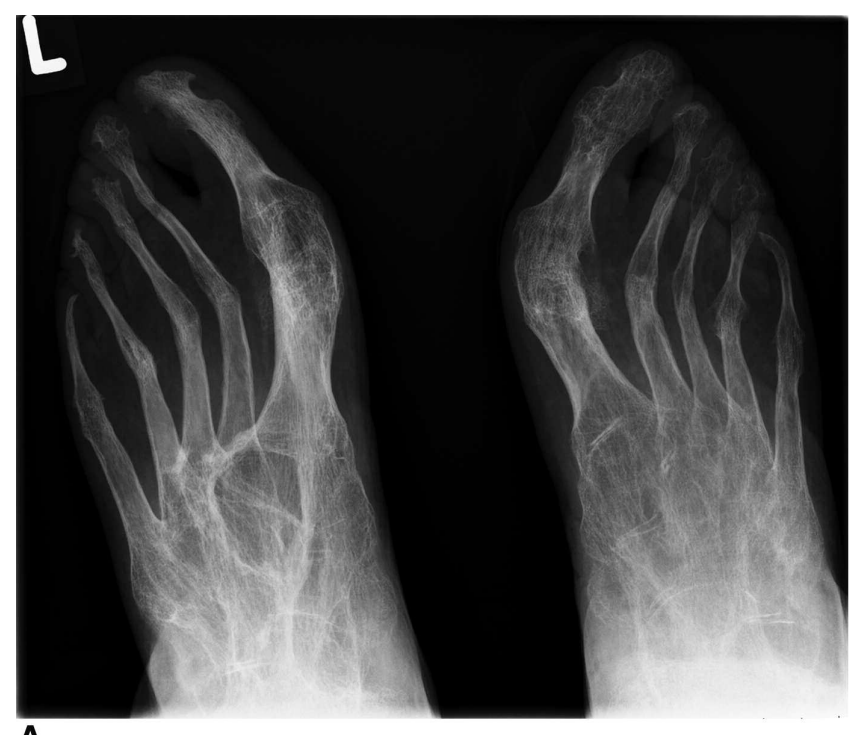

A tations, multiple arthritic deformities, and arthritis. She had pain at the right eye, due to recurring uveitis. Radiographs of the feet showed bilateral ankylosis of all toes and metatarsus (Figure 1). Additionally, she had bilateral ankylosis of sacroiliac joints, and ankylosis of the lower lumbar spine up to the facet joints of the cervical spine (Figure 2). HLA-B27 was negative. Because of active arthritis (predominantly of the ankle and wrists, Figure 3), recurring uveitis, severe plaque psoriasis, and comorbidity renal failure, we initiated immunosuppressive therapy with etanercept.

In the era of powerful disease-modifying antirheumatic drugs and tumor necrosis factor inhibitors, this case provides a radiographic picture of very rare late-stage severe axial and peripheral involvement in an HLA-B27-negative patient with PsA.

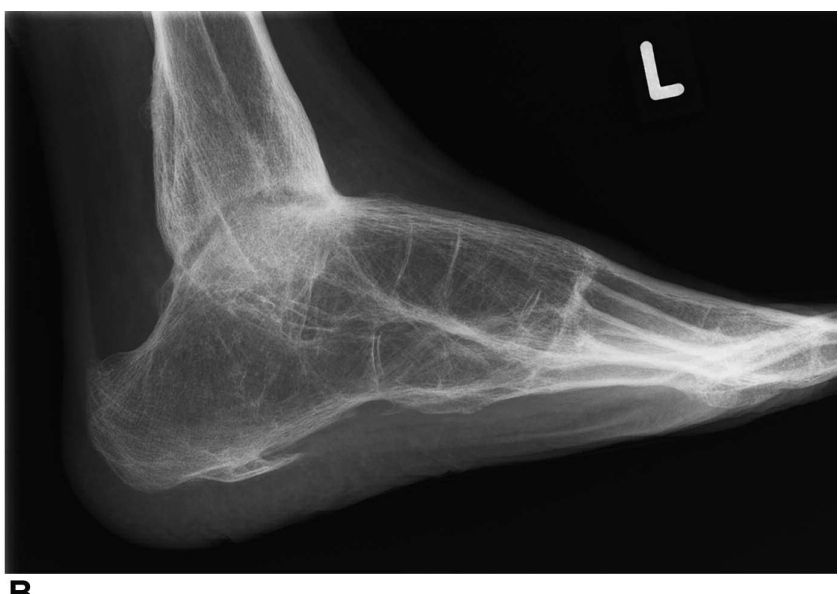

B

Figure 1. A and B. Bilateral complete ankylosis of all toes and metatarsus. 


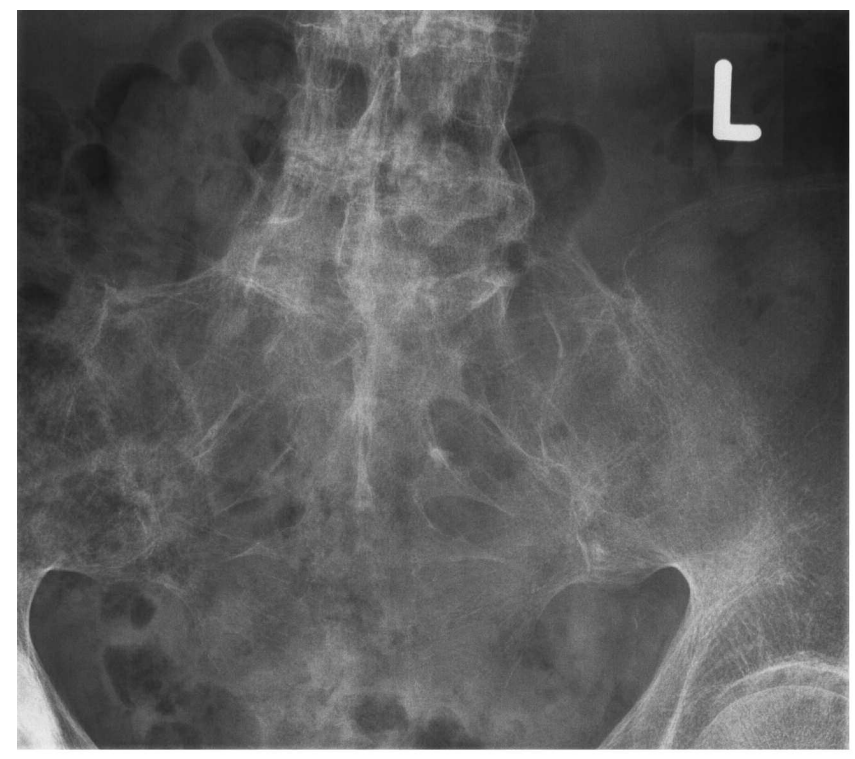

A

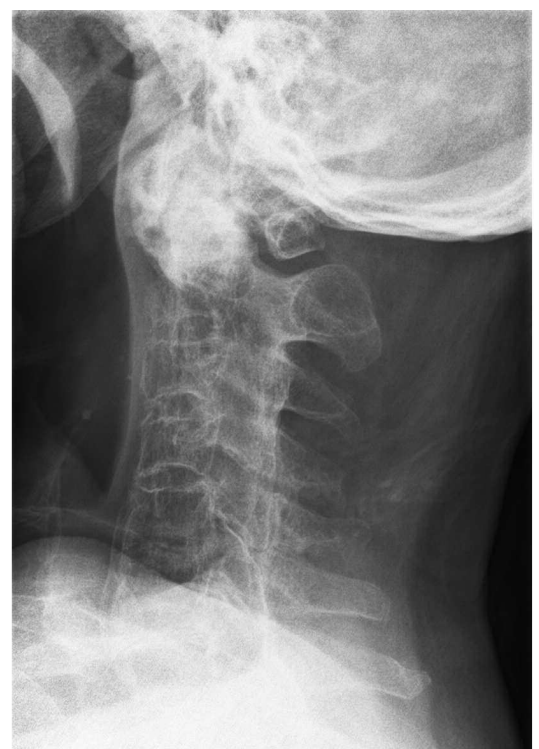

B

Figure 2. A. Bilateral ankylosis of sacroiliac joints and fusion of the lower lumbar spine. B. Syndesmophytes and multisegmental ankylosis of the facet joints of the cervical spine.
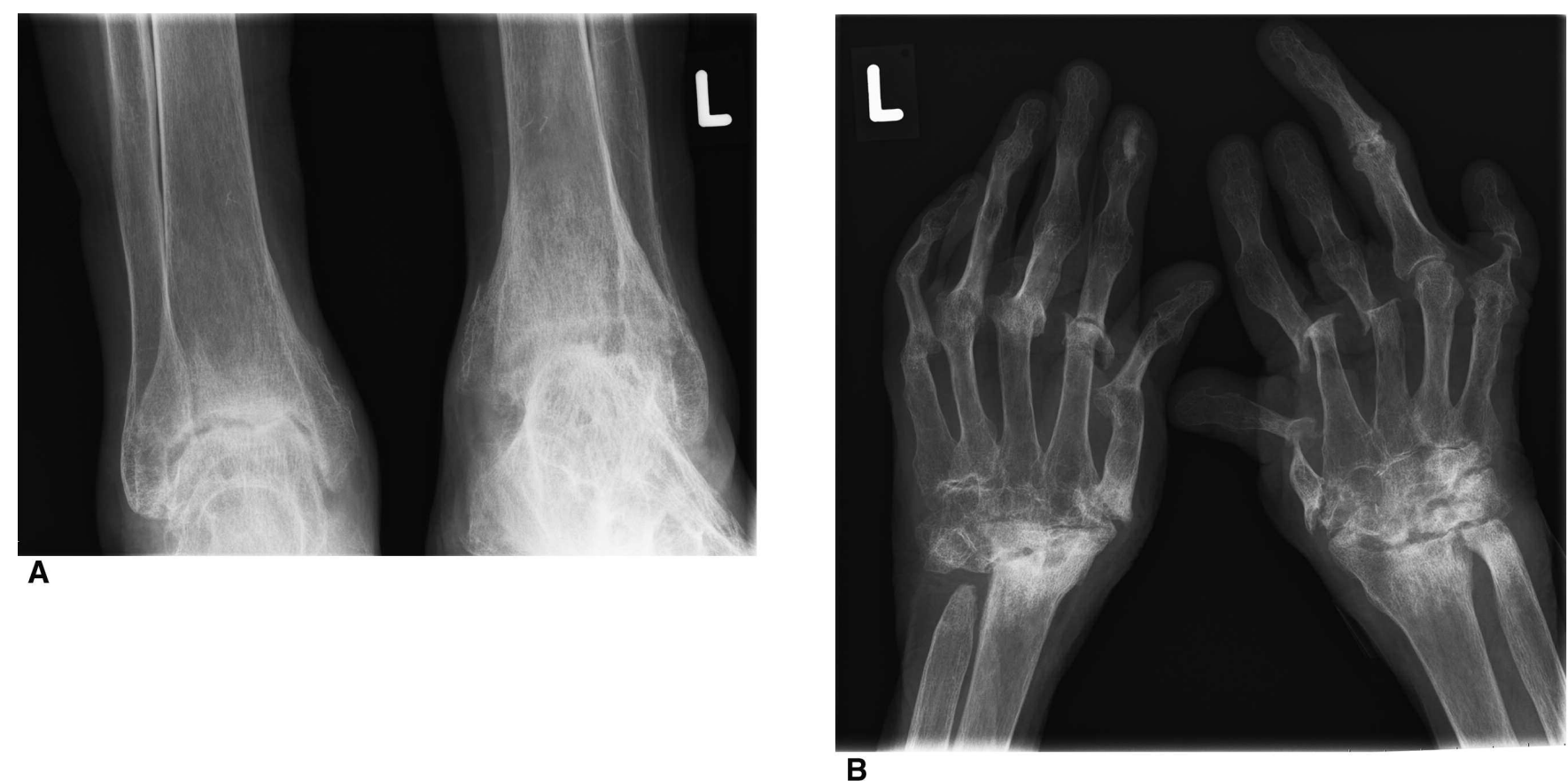

Figure 3. A. Severe bilateral arthritis of the ankle. B. Severe bilateral arthritis of the wrists, ankylosis of multiple proximal interphalangeal (PIP) and distal interphalangeal joints, as well as pencil-in-cup phenomenon of right metacarpophalangeal I and PIP V joints. 